\title{
COMBINATION EFFECT OF LISINOPRIL AND DILTIAZEM AS ANTI FIBROSIS IN PERITONEUM OF RATS
}

\author{
WACHID PUTRANTO ${ }^{1,2}$, IMAM EFFENDI ${ }^{3}$, KUSMARDI KUSMARDI ${ }^{4 *}$, HAMZAH SHATRI $^{3}$ \\ ${ }^{1}$ Student of Doctoral Programme, Faculty of Medicine, Sebelas Maret University, Surakarta, Indonesia. ${ }^{2}$ Department of Internal Medicine, \\ Faculty of Medicine, Sebelas Maret University, Surakarta, Indonesia. ${ }^{3}$ Department of Internal Medicine, Faculty of Medicine, Universitas \\ Indonesia, Jakarta, Indonesia. ${ }^{4}$ Department of Anatomical Pathology, Faculty of Medicine, Universitas Indonesia, Jakarta, Indonesia. \\ Email: kusmardis@gmail.com
}

Received: 15 October 2017, Revised and Accepted: 25 November 2017

\section{ABSTRACT}

Objective: The objective of this research was to evaluate the effect of combination between lisinopril and diltiazem in reducing expression of transforming growth factor- $\beta$ (TGF- $\beta$ ) and fibrosis peritoneum in a male rat treated with continuous ambulatory peritoneal dialysis (CAPD).

Method: This was an experimental study, post-test only control group design. Thirsty Dawley Sprague rats are divided into five groups: Control group (Group 1), CAPD liquid 4,25\% (Group 2), lisinopril 1.44 mg oral and CAPD (Group 3), and diltiazem CD $6.48 \mathrm{mg}$ oral and CAPD (Group 4), lisinopril $1.44 \mathrm{mg}+$ diltiazem CD $6.48 \mathrm{mg}$ oral and CAPD (Group 5). After 4 weeks, rats sacrificed expression of TGF- $\beta$ and peritoneal fibrosis are conducted by histopathology with hematoxylin-eosin staining and immunology with anti-human-TGF- $\beta$.

Result: Twenty peritoneal of rats can be examined. Mean score of TGF- $\beta$ in control group is 1,8 , in CAPD group is 2 , in lisinopril and CAPD group is 1 , 8 , in diltiazem CD and CAPD group is 1,8, in lisinopril and diltiazem CD and CAPD group is 1,7 ( $p=0.959$ ). Mean score of peritoneal fibrosis in control group is 1,1 , in CAPD group is 2, 6, in lisinopril and CAPD group is 1,2 , in diltiazem CD and CAPD group is 1,3 , in lisinopril and diltiazem CD, and CAPD group is $1,5(\mathrm{p}=0,268)$.

Conclusion: Combination of lisinopril and diltiazem lower the expression of TGF- $\beta$ and peritoneum fibrosis better than lisinopril or diltiazem but statistically not significant. Combination of lisinopril and diltiazem lower the peritoneal fibrosis but statistically not significant, and it does not better than lisinopril or diltiazem.

Keywords: Angiotensin converting enzyme inhibitor, Calcium channel blocker, Transforming growth factor- $\beta$, Peritoneal fibrosis.

(C) 2018 The Authors. Published by Innovare Academic Sciences Pvt Ltd. This is an open access article under the CC BY license (http://creativecommons. org/licenses/by/4. 0/) DOI: http://dx.doi.org/10.22159/ajpcr.2018.v11i3.23152

\section{INTRODUCTION}

The final treatment of kidney disease is dialysis. Types dialysis of continuous ambulatory peritoneal dialysis (CAPD) is recently developed, and it is a dialysis method using peritoneum as a semipermeable membrane where diffusion and ultrafiltration (UF) is occurred. The number of patients taking CAPD programs has rapidly increased, especially in some Asian countries. CAPD has a disadvantage as a developing and widely chosen method in the final treatment of kidney disease. One of them is the long-term complication of serious damage to peritoneum membrane [1-3].

Acute and chronic inflammation of peritoneum is the determinant of the beginning and progression of worsening peritoneum membranes. In addition, cytokines and growth factors that are produced during peritoneum inflammation that is transforming growth factor- $\beta$ (TGF- $\beta$ ) is a profibrotic cytokine and is considered to be a main molecule for peritoneal fibrosis. Kyuden et al., [2] have demonstrated the correlation between TGF- $\beta$ of peritoneal fibrosis in vivo at the experimental rat. The formation of advanced glycosylated end product (AGEP) during the CAPD process links to the AGE receptor (RAGE) in the peritoneum membrane, activates the secondary messenger system and increases the production of vascular endothelial growth factor (VEGF) and TGF- $\beta[1,2]$

Duman et al. [4] provided angiotensin converting enzyme inhibitors (ACEI) in albino Wistar rat that had been given $3.86 \%$ intraperitoneal dextrose then, examined the levels of TGF- $\beta$ and peritoneal morphology. The administration of ACEI resulted in a significant reduction of TGF- $\beta$ levels, but the peritoneum fibrosis was not fully inhibited [4] Fang, et al. [5] discovered that the administration of calcium channel blocker (CCB) could suppress the TGF- $\beta$ production and assumed that diltiazem was a potential drug in preventing peritoneal fibrosis [5].

Lisinopril is one of the hypertension drugs in ACEI group while diltiazem is a hypertension drugs in CCB group. They have been widely used as antihypertensive drugs, but their benefits specifically in reducing peritoneal fibrosis were unused. There is no database on the implementation of combinations both lisinopril and diltiazem as peritoneal antifibrosis drugs.

\section{Theoretical background}

At present, CAPD as one of the dialysis method is quite good. It is chosen for children, old and diabetic nephropathy patient. Therefore, the simplicity of the safety from machine less life, comfort feeling, option of the patient become doctor's attraction. This therapy is based on the ability of peritoneal membrane as a dialysis membrane, where there is an exchange process of solutes and toxic products between CAPD and circulating fluid. CAPD fluid is inserted into the peritoneal cavity through a permanent catheter. It contains glucose which facilitates fluid's transfer from the bloodstream to the peritoneal cavity and set to the removal of toxic and aquatic products [6,7].

The working rule of CAPD is diffusion and UF. This is a concept to eliminate azotemia toxins, continuous restore of fluid and electrolyte disturbances throughout the day and unfluctuation such as hemodialysis [8]. 
A serious complication after long-term CAPD is peritoneal fibrosis. It often begins the occurrence of transport dysfunction. Long-term exposure with CAPD fluid shows that peritoneal membrane exhibits loss of mesothelial single layer. It is shown by the increasing of fibroblast proliferation, accumulation of mesothelial collagen and neoangiogenesis, and finally UF failure. TGF- $\beta$ plays a significant role in fibrogenic process at peritoneal membranes. During CAPD, glucose and its breakdown products in CAPD fluid induce local TGF- $\beta$ production. The persistent increasing of TGF- $\beta$ production in dialysis of CAPD patients correlates to an increased risk of peritoneal fibrosis and peritoneal function decline [6-13].

Long-term contact between peritoneum and peritoneal dialysis fluid that is containing high concentrations of glucose will increase the accumulation of AGEP in the peritoneal membrane. This AGE accumulation contributes to the loss of UF capability. Uptake of AGE by peritoneal cells including fibroblasts is administered through the RAGE. Both of dependent and independent receptor processes are responsible for UF deterioration. There are several subclasses of AGE-specific receptors based on their component solubility. Therefore, it may have a different role. The RAGE is RAGE that is involved in intracellular signal of transduction than AGE breakdown. $\mathrm{N}$-carboxymethyl-lysine is a ligand for AGE that increases AGE expression. RAGE on cell surfaces such as RAGE, galectin 3, and macrophage scavenger receptors can be identified in monocytes, endothelial cells, and kidney cells. Nakamura and Niwa [14] found on immunohistochemical examination, the increase of RAGE expression in peritoneal fibroblasts together with intracellular AGE. These findings suggest that AGE is trapped in peritoneal cells through RAGE, and it is followed by cellular reactions and upregulation of various growth factors [15-17].

The peritoneal mesothelium cells produce various cytokines and growth factors. All AGE types are classified in the peritoneum with growth factors, such as TGF- $\beta$, macrophage-colony stimulating factor (M-CSF), and VEGF. The intensity of expression of the growth factor is proportional to the weight of AGE accumulation. TGF- $\beta$ has a main role in fibrogenesis and promotes fibroblast proliferation to increase the expression of Collagen I and III and tissue inhibitor of metalloproteinase. A large number of AGEs are formed during the CAPD process, bind to the RAGE on various cells in the peritoneum. The interaction between AGE and RAGE initiates the activation of the secondary messenger system and increases the production of VEGF and TGF- $\beta$. The increased expression of VEGF intensifies the angiogenesis and vascular permeability. It is followed by the increasing macromolecular leaking and ultimately contributes to UF failure. TGF- $\beta$ increases the proliferation of fibroblasts and production of M-CSF, it also triggers the recruitment and proliferation of macrophages. The macrophages intensify the production of TGF- $\beta$. It triggers hepatocyte growth factors without causing morphological changes and peritoneal fibrosis through proliferation of fibroblasts, collagen production Types 1 and 3 , and tissue inhibitor of metalloproteinase 1 . Thus, increasing TGF- $\beta$ expression induces mesothelial heavy and peritoneal fibrosis and gives the role on the failure of UF [15-17].

AGEP are produced from a non-enzymatic reaction in the hyperglycemia and oxidative states. They are also associated with a high-fat and high-sugar diet. AGE is a main cause of complications of diabetes mellitus such as nephropathy and retinopathy. AGE is presented as a ligand against to RAGE. RAGE is a membrane receptor of immunoglobulin associated with kinase serine protein/threonine. It mediates intracellular signaling pathways with cell proliferation, survival, migration, and invasion, stromal lysis, angiogenesis, and oxidative stress $[18,19]$.

RAGE is expressed as two forms, namely: Full-length and membranebound (fl-RAGE and mRAGE). It is widely expressed on low levels in cells. The highest RAGE content is found in mature pneumocytes lung Type I compared to other cells. It is also expressed in high levels in conditions that attend the inflammation such as vascular, cancer, neurodegeneration, and diabetes $[19,20]$

TGF- $\beta$ is a protein that controls proliferation, cellular differentiation, and other functions in whole cells. TGF- $\beta$ has a role in immunity, cancer, heart disease, diabetes, and Marfan syndrome [21].

Some cells secrete TGF- $\beta$ and also have receptors for TGF- $\beta$. It is known as an autocrine mechanism. Cancer cells increase the production of TGF- $\beta$ that works on nearby cells. TGF- $\beta$ is an expressed protein, and they are classified into three types: TGF- $\beta 1$, TGF- $\beta 2$, and TGF- $\beta 3$ [21]. TGF- $\beta 1$ was first identified in human thrombocyte as a protein with a $25 \mathrm{kD}$ molecular weight with a potential role in wound healing. TGF- $\beta 1$ has a significant role in controlling the immune system, and shows different activities either in different cell types or at different stages of cellular development. Almost immunocompetent cells express TGF- $\beta 1$ [22]. Almost every cell including epithelial, endothelial, hematopoietic cells, nerve cells, and connective tissue cells produce TGF- $\beta$ and has the TGF- $\beta$ receptor. TGF- $\beta$ controls the proliferation cell, differentiation cell, embryonic development, injured healing, and angiogenesis [23].

The peptide structure of three members of the TGF- $\beta$ is very similar. They are all encoded as large protein precursors. TGF- $\beta 1$ contains 390 amino acids while TGF- $\beta 2$ and TGF- $\beta 3$ contain 412 amino acids each. TGF- $\beta$ consists of three types, namely, TGF- $\beta 1$, TGF- $\beta 2$, and TGF- $\beta 3$. Each form is encoded by different genes and messenger ribonucleic acids (mRNA) TGF- $\beta 1$ is expressed by macrophages, endothelial, hematopoietic cells, and histiocyte cells, mRNA TGF- $\beta 2$ in nerve and epithelial cells, whereas mRNA TGF- $\beta 3$ is primarily expressed on mesenchymal cells. The kind of TGF- $\beta$ forms differs from their affinity binding to TGF- $\beta$ receptors $[24,25]$.

Increased or decreased production of TGF- $\beta 1$ is correlated with a number of disease condition. It is included atherosclerosis, glomerulosclerosis and interstitial fibrosis of the kidney, liver, and lungs. TGF- $\beta 1$ mediates kidney hypertrophy, glomerulosclerosis, and tubulointerstitial fibrosis in diabetic kidney disease. TGF- $\beta 1$ is known to be strongly fibrogenic as a result from stimulation of matrix synthesis and inhibition of matrix degradation [26].

Noh, et al. [27] demonstrated the presence of local angiotensin II in human peritoneal mesothelial cells (HPMC). They showed that HPMCs express mRNAs that encode angiotensinogen, ACE, and AT1. HPMC also have the ability to produce angiotensin II. Arranging of local angiotensin II is convinced to trigger the cell growth and extracellular matrix, especially during injured healing. There are correlations between local angiotensin II and TGF- $\beta$ [27]

Diabetes mellitus patients with high glucose levels will increase the AGEP and Advanced Lipoxidation End Product. AGEP stimulates endothelial to express endothelin. It causes vasoconstriction and hypoxia in Apparatus Juxtaglomerular. The Apparatus Glomerular will express renin and finally increase the expression of angiotensin II. Angiotensin II stimulates macrophages to produce TGF- $\beta 1$. Moreover, it stimulates fibroblasts and smooth muscle cells expressing collagen Type I so resulting in interstitial fibrosis [28].

ACE is an enzyme that converts angiotensin I into angiotensin II. The changing of angiotensin I to angiotensin II does not only occur in the lungs, but it is also found in all vascular epithelial tissue [29]. The chain of renin system to angiotensin II is known as the Renin-Angiotensin Aldosterone System [30]. One of the medicines that are used to depress blood pressure in hypertension patient is ACEI. They are the best medicine for cardiovascular disease, especially in improving arterial function and anatomy. It also improves the endothelial function, regress media tunica besides regress and stabilize atherosclerotic plaques [31]. Medicines that are included as ACEI work by inhibiting the effects of angiotensin II as a vasoconstrictor. Angiotensin II is the main peptide that roles in the renin-angiotensin aldosterone system. 
The effect of angiotensin II is mediated by its bond with the angiotensin II receptors, namely: AT1 and AT2. Angiotensin II is considered a growth factor that has a role in cell proliferation, apoptosis, and fibrosis. ACEI that are working with the inhibition of angiotensin II will decrease proteinuria, inflammatory cell infiltration, fibrosis, and growth factor $[4,32,33]$.

CCB inhibits the inclusion of external calcium $(\mathrm{Ca}++)$ ions into cells, so it reduces impulse distribution, myocardial contractions, and blood vessel walls. The most important effects are coronary vasodilatation, peripheral vasodilation, depressing heart activity, and avoiding erythrocyte freezing. Ca-antagonists facilitate natriuresis by increasing kidney blood flow, dilating afferent arterioles and increasing glomerulus filtration pressure. Nondihydropyridine $\mathrm{Ca}$ antagonists (different in dihydropyridine $\mathrm{Ca}$ antagonists) reduces proteinuria by increasing glomerular permeable selectivity to albumin and decreases kidney perfusion pressure. CCB are a group of medicines and often used in the management of hypertension in patients with chronic kidney disease who got CAPD. This medicine has the ability to inhibit matrix production in various cell types. Diltiazem not only reduces the production of collagen but it also reduce production of TGF- $\beta$ by inhibiting the Interleukin-1 $\beta$ (IL-1 $\beta$ ) $[5,34,35]$.

\section{MATERIALS AND METHODS}

\section{Materials}

All the solvents and chemicals required were of analytical grade. Dianeal fluid, salt aquadest, lisinopril dan diltiazem CD was obtained from pharmacy unit of Cipto Mangunkusumo National Hospital, Jakarta.

\section{Experimental animals}

Thirty male white rats (Sprague Dawley) aged 3-4 months with weight 200-300 g were procured from Veterinary Laboratory of Research and Development Centre, Ministry of Health, Jakarta. The animals were maintained under standard conditions of relative humidity and temperature and acclimatized under laboratory conditions before carrying out the experiments. Ethical approval was obtained from The Health Research Ethics Committee, Faculty of Medicine Universitas Indonesia, number 903/UN2.F1/ETIK/2014

\section{Research procedure}

The allocation of experimental animals into each homogenous treatment was done randomly to maintain internal validity [36,37]. Rat is grouped into five groups:

1. Group 1: Rat only get salt aquadest intraperitoneally

2. Group 2: Rat got $4.25 \%$ of Dianeal fluid peritoneally.

3. Group 3: Rat received $4.25 \%$ Dianeal fluid therapy and lisinopril orally.

4. Group 4: Rat received 4.25\% Dianeal fluid therapy and CD diltiazem orally.

5. Group 5: Rat received 4.25\% Dianeal fluid therapy and lisinopril and diltiazem CD orally.

All groups are consisted of six rats and received therapy for 4 weeks, respectively. Rats were sacrificed by cervical dislocations. The peritoneal tissue is examined immunohistochemically for the examination of TGF- $\beta$ expression and histopathologically to determine peritoneal fibrosis.

Initial measurements were not performed because they were the same for all groups from a single population. Thus, the experimental design could be developed without any pretest, but it only used post-test/posttest only control group design [38].

\section{Hematoxylin eosin staining}

The procedures for hematoxylin-eosin staining were in accordance with previous studies [14], in brief, deparaffinized section using xylol then re-hydrated using alcohol and washed in running water. The preparation was inserted into hematoxylin (Meyer solution), rinsed in running water and dipped into a saturated lithium carbonate, after that rinsed with running water for $5 \mathrm{~min}$.

\section{HISTOPATHOLOGY ASSESSMENT RESULT}

Analysis of histopathological changes was made by reading the outward appearance in a blind way and performed by 2 technicians; then the results were averaged. The measurements of TGF- $\beta$ expression were performed by observing the positivity of peritoneal cells, inflammatory cells, and vascularization of immunohistochemical using TGF- $\beta$ antihuman primary antibodies in normal rat peritoneal tissue at negative control and rats that were receiving CAPD in the treatment group [39]. The mesothelial cells of the peritoneum, inflammatory cells, and vascular tissues are expressed positively to express TGF- $\beta$ when they are browned on the cytoplasm and their cell membrane, whereas they are considered as negative if they are blue in the cytoplasm and their cell membrane. All peritoneal tissues that were made in immunohistochemical preparations will be assessed positivity of TGF- $\beta$. Positivity in three components of peritoneum, namely, peritoneal cells, inflammatory cells, and vascularization is added. The result of the sum of positivity is the score that is used as the expression value of TGF- $\beta$.

An assessment of fibrosis on peritoneal tissue was performed on all histopathologic tissues signed with hematoxylin-eosin. The number of fibrosis areas on the entire peritoneal tissue was calculated, and then it was made the scoring system in further processing. The scoring system use is:

- Score 1 , in case of fibrosis in $<25 \%$ of the peritoneal tissue

- Score 2, if fibrosis occurs in $25-50 \%$ of the peritoneum tissue.

- Score 3 , if fibrosis occurs in more than $50 \%$ of the peritoneal tissue.

\section{Statistical analysis}

The values were expressed as a mean standard deviation. $\mathrm{p}<0.05$ was considered significant, denoted by the symbol $\left({ }^{*}\right)$. The data were analyzed by one-way analysis of variance followed by Tukey multiple comparisons using SPSS 20 Software. Inhomogeneous data analyzed nonparametrically by Kruskal-Wallis.

\section{RESULTS}

\section{Expression of TGF- $\beta$}

The expression score of TGF- $\beta$ on tissue peritoneum of control and treatment rat is presented in the following Graphs 1 and 2.

Fig. 1 shows treatment of CAPD using Dianeal fluid $4.25 \%$ increase TGF- $\beta$ expression based on the mean score of the control group of 1.8 compared to the CAPD group of $2.0(\mathrm{p}=0.959)$.

Fig. 2 shows administration of lisinopril, diltiazem, lisinopril+diltiazem tends to decrease TGF- $\beta$ expression as in negative control. Mean score of TGF- $\beta$ negative control group 1.8, CAPD 2 group, CAPD+lisinopril group 1.8, CAPD+diltiazem group 1.8, CAPD+lisinopril+diltiazem group $1.7(\mathrm{p}=0.959)$.

\section{Peritoneal fibrosis}

Figs. 3 and 4 show fibrosis scores of peritoneum in all groups, both of control and treatment groups.

Fig. 3 shows the treatment with CAPD using a 4.25\% Dianeal tends to have a higher mean fibrosis score than the control group. The mean score of the control group was 1.1, and CAPD group was $2.6(\mathrm{p}=0.355)$.

Fig. 4 presents the treatment with lisinopril, diltiazem, lisinopril+diltiazem reducing peritoneal fibrosis. Fibrosis peritoneum score of group CAPD 2.6, ACEI group 1.2, CCB group 1.3, group ACEI+CCB 1.5 uses the treatment of ACEI, CCB, ACEI+CCB reduced peritoneal fibrosis ( $p=0.268$ ). The fibrosis scores of treatment groups with ACE, CCB, and combination of them is higher than the score in the negative control group, but all scores had an average under the CAPD group that did not receive ACEI, CCB and a combination of them. 


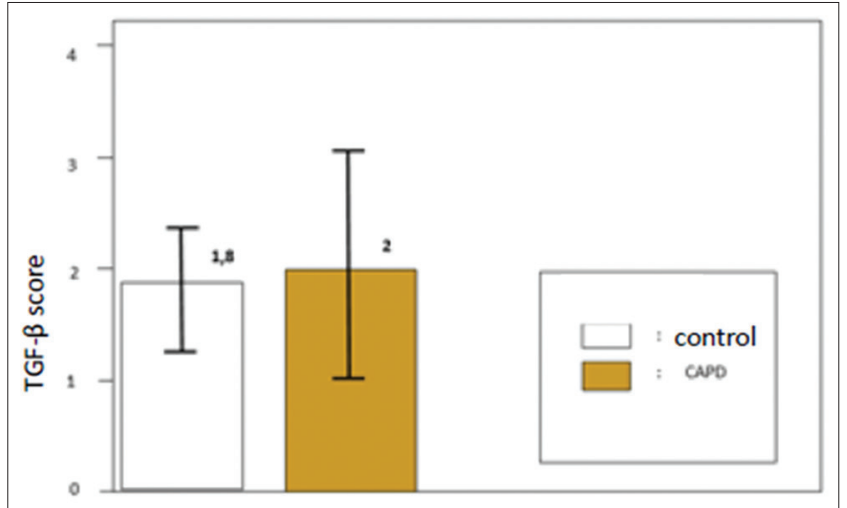

Fig. 1: Expression of transforming growth factor- $\beta$ peritoneum of rat in the control group and continuous ambulatory peritoneal dialysis after 4 weeks

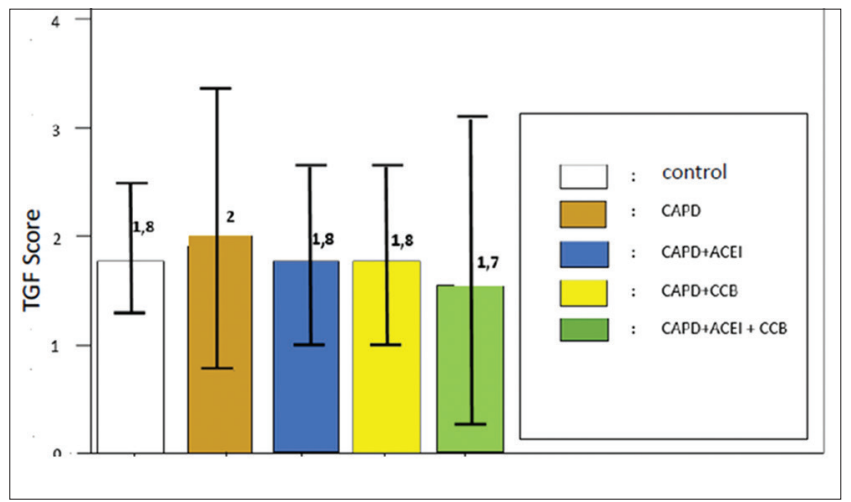

Fig. 2: Expression of transforming growth factor- $\beta$ peritoneal rat in the control group, continuous ambulatory peritoneal dialysis group, angiotensin converting enzyme (ACE) inhibitors, calcium channel blocker (CCB), and ACE+CCB combination after 4 weeks

\section{DISCUSSION}

\section{TGF- $\beta$ expression and fibrosis peritoneum}

The identification of mechanisms that are involved in pathogenesis of peritoneal membrane failure during the long CAPD process is still a challenge. Fibroblasts and inflammatory cells are classically considered to be the primary cells that respond to the changes in the structure and function of the peritoneum. Acute and chronic inflammations are convinced to be the main factor in changing the structures and functions. Among the cytokines and growth factors produced during peritoneal inflammation, TGF- $\beta$ is a strong pro-fibrotic cytokine and considered as main molecule in the peritoneal fibrosis process. As the expression of TGF- $\beta$, some pro-inflammations such as TNF- $\alpha$, IL-1, IL8 , and other pro-fibrotic cytokines like angiotensin II show a role in peritoneal fibrosis [1].

The objective of this study was to determine whether the combination of ACEI and CCB can decrease the expression of TGF- $\beta$ and reduce peritoneal fibrosis better than ACEI or CCB as a single medicine. This study result told us that combination of lisinopril and diltiazem could decrease TGF- $ß$ expression in Dawley Spraque rat, but it is not significant $(p=0.555)$. The decrease of TGF- $\beta$ expression in the combination group has a lower tendency than lisinopril or diltiazem group as a single medicine, but it is not statistically significant $(p=0.959)$. The combination of lisinopril and diltiazem may reduce peritoneal fibrosis of Dawley Spraque rat, but it is not statistically significant ( $p=0.268$ ). The combination of both drugs in reducing peritoneal fibrosis of Dawley Spraque rat is not better than lisinopril and diltiazem as a single medicine $(\mathrm{p}=0.355)$.

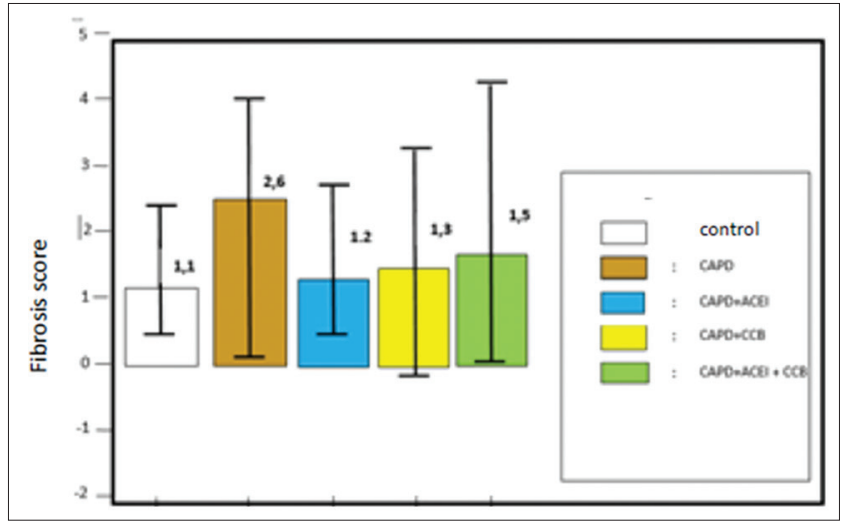

Fig. 3: The average peritoneal fibrosis scores of rat in the control and continuous ambulatory peritoneal dialysis group after 4 weeks

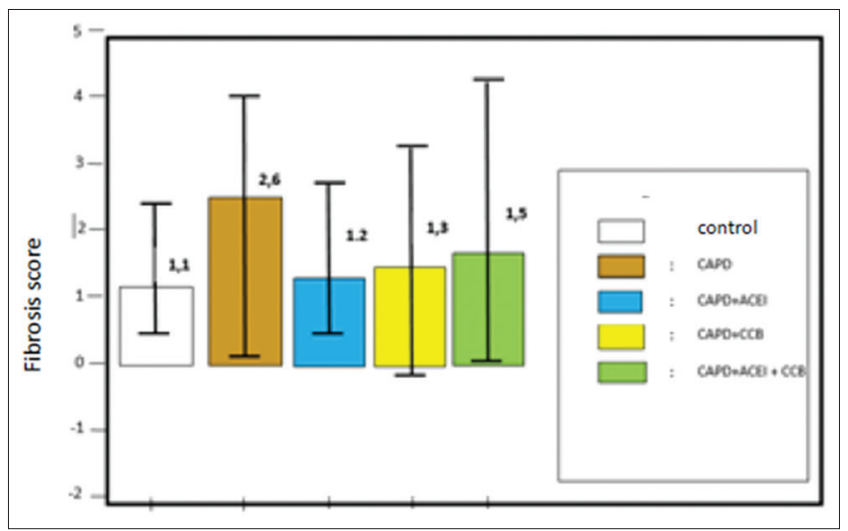

Fig. 4: The mean score of fibrosis peritoneal tissue of rat in the control group, CAPD group, angiotensin-converting enzyme inhibitors (ACEI), calcium channel blocker (CCB), ACEI+CCB after 4 weeks

In the Study of Duman et al., [40] the used of lisinopril in peritoneal fibrosis of rat showed that there was a significant decrease in TGF- $\beta$ expression $(\mathrm{p}<0.05)$, while on histologic examination showed peritoneum fibrosis reduction compared to dextrose group but it was not statistically significant ( $\mathrm{p}>0.05)$. In another study, Duman et al. [4] used another ACEI like enalapril, obtained high TGF- $\beta$ results in the CAPD group, undetectable in the control group and CAPD+enalapril $(\mathrm{p}=0.01$ ) fibroblast peritoneum decreased in CAPD+enalapril compared to CAPD $(\mathrm{p}>0.05)$.

The mechanism by which the peritoneal fluid of hypertonic dialysis induces structural changes of the peritoneum is not fully understood. In this study, we show that high glucose concentrations increased the expression of TGF- $\beta$. This is consistent to the previous research that TGF- $\beta$ has a role in mediating the changes on the peritoneal membranes as high-glucose dialysate. Injury in peritoneum is mediated by angiotensin II and correlated with stimulation of AT receptor I $[4,40]$.

Histopathologically, Duman et al. $[4,40]$ got less cell infiltration in the therapeutic group and inhibition of angiotensin II blocker receptors only explain the part of this condition. Angiotensin II blocker receptors reduce injury to high glucose dialysate fluid by inhibiting excessive cytokine expression such as TGF- $\beta$, and it maintains peritoneal viability in CAPD patients over a long-term period [4.40].

Kyuden et al. [2] presented their research that TGF- $ß$ expression and peritoneal cell proliferation did not return to control levels after the addition of ACEI, ARB, or combination of them. The addition of these 
drugs leads to complete the inhibition of TGF - $\beta$ expression, but it does not completely prevent in changing peritoneal fibrosis. Research conducted by Duman et al. [40] using ACEI and Kyuden et al. [2] using ACEI and ARB showed that TGF- $ß$ may one of peritoneal fibrosis factor.

Research conducted by Fang et al. [5] in vitro using omentum gastric tumor patients who have received gastrectomy presented diltiazem decrease TGF $\beta$ significantly ( $p>0.05$ ). It decreases fibrosis by inhibiting collagen Types I and III. The fibrosis assessment of Fang did not assess fibrosis using histopathologic features but collagen Types I and III.

The combination of lisinopril and diltiazem has a tendency to decrease the expression of TGF- $ß$ better than lisinopril and diltiazem as a single medicine, but this combination cannot repair the fibrosis. This is due to the fact that both drugs are equally antifibrosis works against TGF- $\beta$. Either lisinopril or diltiazem cannot reduce fibrosis by improving fibrinolysis processes through inhibition of tissue plasminogen activator (t PA) [1].

The TGF- $\beta$ is a complex way and involves a variety of other mediators. Regulatory activation of TGF- $\beta$, intracellular signaling, and interaction of DNA transcription factors initiate a change in response to TGF- $\beta$. Other cytokines have the ability to alter TGF- $ß$ response to fibrosis. IL-1 and IL- 6 are mediators that capable to alter TGF- $\beta$ response [41-43].

Dhodi et al. [42] reported that the use of Irbesartan and spironolactone in reducing peritoneal fibrosis resulted in TGF- $\beta$ levels decreased significantly on the irbesartan and spironolactone groups, but the combination of them did not significantly decrease peritoneal fibrosis. Peritoneal fibrosis and inflammatory were reduced in three groups, but it was not statistically significant. This is due to the fact that limited duration of the study.

The pathological change mechanism occurring in the CAPD peritoneum is not fully understood. However, it is suspected that there is involvement of mesothelial peritoneum, macrophage, and fibroblasts in the inflammatory response of the peritoneal membrane. They also have a role in the formation of extracellular matrix and remodeling through growth factors and cytokines [44].

Maintaining the function of the peritoneum is a main step taken to ensure CAPD process effectiveness over the long-term. Several steps can be taken to make the peritoneal membrane work properly, and solute regulation and water transport improve peritoneal microcirculation by increasing vasodilator secretion, such as prostaglandin E2 and nitric oxide, peritoneal fibrinolysis regulation, extracellular production, and remodeling matrix, and mechanism local antibacterial defense in the peritoneum $[4,42]$.

Drop out incidence in this study is quite much either dead rat or due to the failure of peritoneal taking since the thin peritoneum. Drop out obtained 2 rat died due to predation and 8 failure in taking peritoneum for making preparations of TGF- $ß$ and peritoneal fibrosis. It resulted in the calculated size of the sample being too small, so it causes the statistical analysis used is non-parametric analysis. Trough nonparametric analysis, the researcher, cannot observe the difference of TGF- $\beta$ expression change and the peritoneal fibrosis of the combination group of lisinopril and diltiazem with the lisinopril or diltiazem group given separately. Since this non-parametric analysis is not sensitive to differentiate the small mean.

\section{Limitation of the study}

This study had several limitations: (1) The difficulty determines appropriate dose from diltiazem in reducing peritoneal fibrosis since there are no prior studies conducted on experimental animals. (2) CAPD conducted in experimental animals in the form of concentrated dioxide fluid injections may be less able to describe the condition of CAPD in humans. It is due to CAPD carried out catheter, and dialysis fluids are given continuously with fluid replacement every 6 h. (3) The researcher did not conduct other pro-inflammatory cytokine tests that may affect TGF- $\beta$ response in peritoneal fibrosis.

\section{CONCLUSION AND SUGGESTION}

\section{Conclusion}

1. The combination of lisinopril and diltiazem has a tendency to decrease TGF- $\beta$ expression in rat peritoneum by CAPD, and this combination tends to be better than lisinopril or diltiazem as a single drug.

2. The combination of lisinopril and diltiazem has a tendency to reduce fibrosis in peritoneum rat by CAPD, but this combination is not better than lisinopril and diltiazem as a single drug.

\section{Suggestion}

1. The advanced study is taking into account the effects of IL- 1 and IL-6 in assessing the response changes of TGF- $ß$ to fibrosis.

2. The advanced research using a combination of ACE/CCB with other medicine that may work as fibrinolysis (e.g., simvastatin) by inhibition of TPA.

\section{AUTHORS CONTRIBUTION}

Wachid Putranto: Carried out the experiment, conceived the original idea. Imam Effendi: Supervise the project. Kusmardi Kusmardi: Carried out the experiment, wrote the manuscript. Hamzah Shatri: Wrote the manuscript.

\section{CONFLICT OF INTEREST}

The authors declare no conflict of interest, financial or otherwise.

\section{REFERENCES}

1. Aroeira LS, Aguilera A, Sánchez-Tomero JA, Bajo MA, del Peso G, Jiménez-Heffernan JA, et al. Epithelial to mesenchymal transition and peritoneal membrane failure in peritoneal dialysis patients: Pathologic significance and potential therapeutic interventions. J Am Soc Nephrol 2007; 18:2004-13.

2. Kyuden Y, Ito T, Masaki T, Yorioka N, Kohno N. Tgf-beta1 induced by high glucose is controlled by angiotensin-converting enzyme inhibitor and angiotensin II receptor blocker on cultured human peritoneal mesothelial cells. Perit Dial Int 2005;25:483-91.

3. Del Peso G, Jiménez-Heffernan JA, Bajo MA, Aroeira LS, Aguilera A, Fernández-Perpén A, et al. Epithelial-to-mesenchymal transition of mesothelial cells is an early event during peritoneal dialysis and is associated with high peritoneal transport. Kidney Int Suppl 2008;108:S26-33.

4. Duman S, Günal AI, Sen S, Asçi G, Ozkahya M, Terzioglu E, et al. Does enalapril prevent peritoneal fibrosis induced by hypertonic (3.86\%) peritoneal dialysis solution? Perit Dial Int 2001;21:219-24.

5. Fang CC, Yen CJ, Chen YM, Chu TS, Lin MT, Yang JY, et al. Diltiazem suppresses collagen synthesis and IL-1beta-induced TGFbeta1 production on human peritoneal mesothelial cells. Nephrol Dial Transplant 2006;21:1340-7.

6. Schilte MN, Celie JW, Wee PM, Beelen RH, van den Born J. Factors contributing to peritoneal tissue remodeling in peritoneal dialysis. Perit Dial Int 2009;29:605-17.

7. Sukandar E. Clinical Nephrology. Bandung: Faculty of Medicine, Pajajaran University; 2006. p. 465-529.

8. Sculman G, Himmelfarb J. Hemodialysis. In: Brenner B, Braunwald E, Fauci AS, editors. The Kidney. $7^{\text {th }}$ ed. Philadelphia, PA: Saunders Elseviers; 2004. p. 1663-8, 19610.

9. Kolesnyk I, Dekker W, Noordzij M. Impact of ACE inhibitors and all receptors blockers on peritoneal membrane transport characteristics in long term peritoneal dialysis patients. Perit Dial Int 2007;27:446-53.

10. Kitiyakara C, Yamwong S, Vathesatogkit P, Chittamma A, Cheepudomwit S, Vanavanan S, et al. The impact of different GFR estimating equations on the prevalence of CKD and risk groups in a southeast asian cohort using the new KDIGO guidelines. BMC Nephrol 2012;13:1.

11. Cohen EP. Chronic renal failure and dialysis. ACP Medicine. Vol. 3. New York: WebMD; 2007. p. 123-6.

12. Ross E, Carusso J. Hemodialysis and continous therapies. In: Daugirdas T, 
Blake G, editors. Handbook of Nephrology and Hypertension $5^{\text {th }}$ ed. Philadelphia, PA: Lippicott Williams \& Wilkins; 2005. p. 281-93.

13. Singh A, Brenner B. Dialysis in the treatment of renal failure. In: Kasper DL, Fauci AS, Longo DL, Braunwald E, Hauser SL, Jameson JL, editors. Harrison Principles of Internal Medicine $16^{\text {th }}$ ed. New York: McGraw-Hill Companies Inc; 2006. p. 1663-8.

14. Nakamura S, Niwa T. Advanced glycation end-products and peritoneal sclerosis. Semin Nephrol 2004;24:502-5.

15. Guo H, Leung JC, Lam MF, Chan LY, Tsang AW, Lan HY, et al. Smad7 transgene attenuates peritoneal fibrosis in uremic rats treated with peritoneal dialysis. J Am Soc Nephrol 2007;18:2689-703.

16. Margetts PJ, Bonniaud P. Basic mechanisms and clinical implications of peritoneal fibrosis. Perit Dial Int 2003;23:530-41.

17. Kihm LP, Müller-Krebs S, Klein J, Ehrlich G, Mertes L, Gross ML, et al. Benfotiamine protects against peritoneal and kidney damage in peritoneal dialysis. J Am Soc Nephrol 2011;22:914-26.

18. Goldin A, Beckman JA, Schmidt AM, Creager MA. Advanced glycation end products: Sparking the development of diabetic vascular injury. Circulation 2006;114:597-605.

19. Cheng L, Tang Y, Zheng D. Advanced glycation end-products activate the renin angiotensin system through the RAGE/P13-K signaling pathway in podocytes. Clin Ivest Med 2012;35:E282-93.

20. Basta G, Schmidt AM, De Caterina R. Advanced glycation end products and vascular inflammation: Implications for accelerated atherosclerosis in diabetes. Cardiovasc Res 2004;63:582-92.

21. Hill JJ, Tremblay TL, Cantin C, O'Connor-McCourt M, Kelly JF, Lenferink AE, et al. Glycoproteomic analysis of two mouse mammary cell lines during transforming growth factor (TGF)-beta induced epithelial to mesenchymal transition. Proteome Sci 2009;7:2.

22. Letterio JJ, Roberts AB. Regulation of immune responses by TGF-beta. Annu Rev Immunol 1998:16:137-61.

23. Blobe G, Schiemann W, Lodish H. Role of transforming growth factor $B$ in human disease. N Engl J Med 2000;343:228.

24. Daopin S, Piez KA, Ogawa Y, Davies DR. Crystal structure of transforming growth factor-beta 2: An unusual fold for the superfamily. Science 1992;257:369-73

25. Taipale J, Saharinen J, Keski-Oja J. Extracellular matrix-associated transforming growth factor-beta: Role in cancer cell growth and invasion. Adv Cancer Res 1998:75:87-134.

26. Chen S, Jim B, Ziyadeh FN. Diabetic nephropathy and transforming growth factor-beta: Transforming our view of glomerulosclerosis and fibrosis build-up. Semin Nephrol 2003;23:532-43.

27. Papagianni A, Kalovoulos M, Kirmizis D, Vainas A, Belechri AM, Alexopoulos E, et al. Carotid atherosclerosis is associated with inflammation and endothelial cell adhesion molecules in chronic haemodialysis patients. Nephrol Dial Transplant 2003;18:113-9.
28. Pennison $\mathrm{M}$, Pasche B. Targeting transforming growth factor- $\beta$ signaling. Curr Opin Oncol 2008;19:579-85.

29. Oates J, Brown N. Antihypertensive agents and drugs therapy of hypertension In: Hardman JG, Gilman AG, editors. The Pharmacological Basis of Theurapeutics. 10 $0^{\text {th }}$ ed. New York: McGraw-Hill; 2001. p. 336-42.

30. Kostova E, Jovanoska E, Zafirov D, Jakovski K, Maleska V, SlaninkaMiceska M, et al. Dual inhibition of angiotensin converting enzyme and neutral endopeptidase produces effective blood pressure control in spontaneously hypertensive rats. Bratisl Lek Listy 2005;106:407-11.

31. Klabunde R. Cardiovasculary Physiology Concepts. Available from: http://www.cvphysiology.com/Blood/20 Pressure/BP001.htm. [Last cited on 2007 Nov 07].

32. Taddei S, Virdis A, Ghadom L, Sudono I, Salvetti A. Effects anti hypertensive drugs on endothelial dysfunction. Drugs 2002;62:265-84.

33. Hajjar I, Kotchen TA. Trends in prevalence, awareness, treatment, and control of hypertension in the united states, 1988-2000. JAMA 2003;290:199-206

34. Sica D. Patophysiologi. In: Black HR, Elliot WJ, editors. Hypertension: A companion to Braunwald's heart disease. $1^{\text {st }}$ ed. New York: WB Saunders Company; 2007. p. 239-53.

35. Kaplan NM.Treatment of hypertention: Drugs therapy. In: Neal W, editor. Kaplan's Clinical Hypertension. 10 ${ }^{\text {th }}$ ed. Philadelphia: Lippincott Williams \& Wilkins; 2010. p. 198-210.

36. Supranto J. Sampling Technique for Survey and Experiment. Jakarta: PT Rineka Cipta; 2007.

37. Kuntoro. Introduction for Sampling Techniques. Surabaya: Directorate of Research Airlangga University; 1994.

38. Zainuddin M. Research Design. Surabaya: Directorate of Research Airlangga University; 1999.

39. Tomino Y. Enzyme Antibody Method. In Laboratory Techniques in Renal Cell and Molecular Biology. Tokyo, Japan: Bunkodo Co, Ltd; 2000. p. 82-93.

40. Duman S, Sen S, Duman C, Oreopoulos DG. Effect of valsartan versus lisinopril on peritoneal sclerosis in rats. Int $\mathrm{J}$ Artif Organs 2005;28:156-63.

41. Bodenham T, Topley N, Fraser D. Peritoneal fibrosis is mouse strain dependent. Nephrol Dial Transplant 2012;27:1-3.

42. Dhodi JB, Mestry SN, Juvekar AR. Diabetic nephropathy-genesis, prevention and treatment. Int J Phar Pharm Sci 2014;6:42-7.

43. Kandavelu S, Somasundaram PC, John B, Rajendran R. Proinflammatory cytokines elicit inflammatory response in blood leukocytes of post dialytic chronic renal patients through heme oxygenase-1 activation. Int J Pharm Pharm Sci 2014;6:111-5.

44. Ersoy R, Celik A, Yilmaz O,Sarioglu S, Sis B, Akan P, et al. The effects of irbesartan anf spironolactone in prevention of peritoneal fibrosis in rats. Perit Dial Int 2007;27:4 